\title{
Cloning and Characterization of the Human USP22 Gene Promoter
}

\author{
Jianjun Xiong ${ }^{1,2}$, Xiangxin Che ${ }^{1,2}$, Xueqin $\mathrm{Li}^{1}$, Huan $\mathrm{Yu}^{1}$, Zhen Gong ${ }^{1}$, Weidong $\mathrm{Li}^{2 *}$ \\ 1 College of Basic Medical Science, Jiujiang University, Jiujiang, China, 2 Key Laboratory of Jiangxi for the Systems Bio-medicine, Jiujiang, China
}

\begin{abstract}
Ubiquitin-specific processing enzyme 22 (USP22) plays a direct role in regulating cell cycle, and its overexpression has been reported to be involved in tumor progression. However, little is known about the regulation of USP22 transcription. In this study, we cloned and characterized the human USP22 promoter. Using 5' RACE (rapid amplification of cDNA ends) analysis, the transcriptional initiation site was identified. Promoter deletion analysis showed that the sequence between -210 and -7 contains the basal promoter for USP22 in human fibroblast and tumor cells. Surprisingly, mutations in a putative Sp1 binding site immediately upstream of the USP22 transcriptional start site $(-13$ to -7$)$ resulted in a significant induction of promoter activity. Further study revealed that Sp1 binds to this site in human normal fibroblast cells, and treatment with the Sp1 inhibitor mithramycin A led to a marked increase in USP22 transcript levels. Forced expression of exogenous Sp1 repressed the USP22 promoter activity in HeLa cells. In contrast, knockdown of Sp1 enhanced USP22 promoter activity and mRNA levels. These data suggest that Sp1 is a crucial regulator of USP22 transcription.
\end{abstract}

Citation: Xiong J, Che X, Li X, Yu H, Gong Z, et al. (2012) Cloning and Characterization of the Human USP22 Gene Promoter. PLoS ONE 7(12): e52716. doi:10.1371/ journal.pone.0052716

Editor: Szabolcs Semsey, Niels Bohr Institute, Denmark

Received August 15, 2012; Accepted November 20, 2012; Published December 26, 2012

Copyright: (c) 2012 Xiong et al. This is an open-access article distributed under the terms of the Creative Commons Attribution License, which permits unrestricted use, distribution, and reproduction in any medium, provided the original author and source are credited.

Funding: This work was supported by the National Natural Sciences Foundation of China (31000581); Natural Science Foundation of Jiangxi, China (2010GQY0199); Jiangxi Science and Technology Support Programmer, China (2010BSA14000); and Science Programmer of Jiangxi Provincial Department of Education, China (GJ11619). The funders had no role in study design, data collection and analysis, decision to publish, or preparation of the manuscript.

Competing Interests: The authors have declared that no competing interests exist.

* E-mail: Iwd62518@yahoo.com.cn

\section{Introduction}

Ubiquitin-specific processing enzyme 22 (USP22), also named USP3L or KIAA1063) is a novel deubiquitinating enzyme, which cleaves ubiquitin (Ub) from Ub-conjugated protein substrates [1]. In eukaryotes, USP22 functions as an enzymatic subunit of the hSAGA transcriptional cofactor complex [2]. It has been documented that a key regulator of cell cycle, $p 21$, is regulated by USP22 [3], and depletion of USP22 results in a G1 phase cellcycle arrest in human tumor cells [2]. Furthermore, USP22 participation in regulating shelterin protein turnover and telomere maintenance has also been demonstrated [4].

In humans, the USP22 gene is located on chromosome 17 and consists of 14 exons [1]. Northern blot analyses demonstrated that USP22 was expressed moderately in various tissues, including heart, skeletal muscle, and weakly in lung and liver [1]. Mitogen activation or virus infection in normal $\mathrm{T}$ and $\mathrm{B}$ lymphocytes may stimulate USP22 expression to promote cell immortalization [5], suggesting that the regulation of USP22 gene expression occurs mainly at the transcriptional level. More importantly, USP22 is considered as one of the putative cancer stem cell markers [2], and changes in the expression of USP22 have been linked with metastatic potential and therapeutic outcome in human cancer $[6,7]$. However, the mechanisms leading to USP22 transcriptional activation, particularly in human tumor cells, are still unknown.

In this article, we tried to analyze USP22 gene regulation at the transcriptional level. By generating a number of $5{ }^{\prime}-$ and $3^{\prime}-$ deletion constructs to delineate the promoter region, we identified a 203-bp fragment necessary for basal transcriptional activity. In addition, we provided evidence for the first time that $\mathrm{Spl}$ is involved in the regulation of human USP22 expression.

\section{Materials and Methods}

\section{Cell cultures}

Normal human lung fibroblast (HFLl) cells and the human cervical cancer cell line (HeLa) were from the American Tissue Culture Collection (ATCG) and were cultured in Dulbecco's modified Eagle's medium (DMEM, Invitrogen) supplemented with $10 \%$ fetal bovine serum (GIBCO) at $37^{\circ} \mathrm{C}$ in a $5 \% \mathrm{CO}_{2}$ and $95 \%$ air in an incubator.

\section{5' Rapid amplification of cDNA ends (5'-RACE)}

A 5'-RACE system was performed according to the manufacturer's instructions (TaKaRa Bio). Briefly, $2 \mu \mathrm{g}$ of total RNA extracted from HeLa cells was treated with calf intestinal phosphatase to remove the free $5^{\prime}$-phosphate group. Tobacco acid pyrophosphatase was then used to specifically remove the cap structure from the full-length mRNA, leaving a 5'-monophosphate. A RNA oligonucleotide adaptor was next ligated to the newly decapped mRNA by T4 RNA ligase. With the ligated RNA as a template, USP22 cDNA was synthesized by reverse transcription using M-MLV reverse transcriptase and random primers. The resulting cDNA was then amplified by nested PCR using LA Taq DNA polymerase as well as the human USP22 gene primer (reverse) and the adaptor primer (forward) provided by the manufacturer. The gene-specific antisense inner primer $5^{\prime}$ GGGGAGGTTCTGCTTCGAGTTGT-3' (+117 to +95$)$ and 
outer primer 5'-CTCGTCGTTGGGGATTATTT-3' (+393 to +374 ) were complementary to the USP22 cDNA sequence. The PCR products were analyzed on agarose gels and cloned into the pMD-18T vector (TaKaRa) for sequencing to determine the transcriptional start site(s).

\section{Genome DNA isolation and cloning of the human USP22 promoter}

Genome DNA was isolated from the HeLa cells using a DNA extraction kit (QIAGEN) and dissolved in water. Based upon our findings regarding the location of the transcriptional start site, a 2880-bp fragment from -2828 to +52 in the $5^{\prime}$-flanking region of the USP22 gene was generated by PCR. The amplified DNA was cloned into a pMD 18-T simple vector and verified by direct sequencing. Other deletion fragments were generated by PCR using this plasmid DNA as a template (see Table 1 for PCR primer sequences). The PCR products were gel-purified, digested with $\mathrm{KpnI}$ and BglII, and subcloned into the pGL3-basic firefly luciferase vector (Promega). All the sequences of the cloned promoter region were confirmed by DNA sequencing. The sequence of the USP22 promoter was analyzed for the presence of consensus transcription factor binding sites using the MatInspector program (Genomatix Software $\mathrm{GmbH}$ ).

Site-directed mutagenesis to inactivate the Spl-binding sites at positions -13 to -7 of the promoter were carried out within the p-210/+52 construct according to the MutanBEST Kit methodology (TaKaRa Bio) with the following primers: mutant Spl site forward, 5'-GATCGGTGCCTGCGTTGCA-3'; deletion Sp1 site forward, 5'-TGCGTGCGTTGCAGCGTCGC-3'; Spl reverse, 5'-CGGAGCTGGGGCTGCTGCGGA-3'. All mutations were confirmed by DNA sequencing.

\section{Transfections and dual luciferase reporter assay}

HFL1 cells and HeLa cells were plated in 24-well plates 24 hours before transfection with $0.5 \mu \mathrm{g}$ of various USP22 promoter constructs and $0.1 \mu \mathrm{g}$ of pRL-TK (Promega) using Lipofectamine 2000 (Invitrogen) in each well. All transfection experiments were repeated five times. Twenty-four hours after transfection, cells were washed in phosphate-buffered saline and lysed for $30 \mathrm{~min}$ at room temperature using passive lysis buffer (Promega). Luciferase activity was determined using the dual luciferase reporter assay system (Promega). Normalized luciferase

Table 1. Primers used in the generation of promoter luciferase constructs.

\begin{tabular}{|c|c|c|}
\hline Construct & Primer & Sequence $5^{\prime} \rightarrow \mathbf{3}^{\prime}$ \\
\hline$p-2880 /+52$ & sense- 2880 & AGGTACCGATAGGGTTTCATCACATTG \\
\hline $\begin{array}{l}p-2880 / \\
-1306\end{array}$ & antisense-1306 & GGAGATCTTGTGGCCAAGACAAATTGCC \\
\hline$p-1306 /+52$ & sense-1306 & AAAGCTITATCCCAGTCGTCAGTCC \\
\hline$p-866 /+52$ & sense-866 & CGGTACCTITGACTTTATTGGGTTGAG \\
\hline$p-866 /-326$ & antisense-326 & GGAGATCTGGCTCTCAGTATAGTCCGTC \\
\hline$p-595 /+52$ & sense-595 & AGGTACCCTGCAAACAGCTCCCGATTA \\
\hline$p-326 /+52$ & sense-326 & CGGTACCTATGACAATAGCCGAAGGTG \\
\hline$p-210 /+52$ & sense-210 & AGGTACCGTCTACCCAGAGCCTAACGG \\
\hline$p-7 /+52$ & sense-7 & AGGTACCTGCCTGCCTTGCAGCCTCCC \\
\hline common & antisense +52 & GGAGATCTGCGGAGGCCGGACAAAGATGGG \\
\hline
\end{tabular}

activity was expressed as the ratio of firefly luciferase activity to Renilla luciferase for each sample.

\section{Chromatin Immunoprecipitation (ChIP) Assay}

ChIP assays were performed according to the EZ-ChIP Kit (Millipore) manufacturer instructions. Briefly, HFL1 cells were fixed by adding formaldehyde to a final concentration of $1 \%$ and incubated by modest shaking for $30 \mathrm{~min}$ at room temperature. Thereafter, cells were washed twice with cold phosphate-buffered saline. The pellet was resuspended and lysed, and nuclei were isolated and sonicated until the chromatin had an average length of 500-1500 bp. After centrifugation, the supernatant was incubated with $3 \mu \mathrm{g}$ of antibody against $\mathrm{Spl}$ overnight at $4^{\circ} \mathrm{C}$ for immunoprecipitation. The following day, magnetic protein-G beads were added and the mix was further incubated at $4^{\circ} \mathrm{C}$ for 1 hour. After appropriate washing, the antibody-transcription factor-DNA complex was eluted from the beads, formaldehyde cross-links were reversed, and proteins were digested with proteinase $\mathrm{K}$ at $67^{\circ} \mathrm{C}$ overnight. DNA was purified and used for PCR with primers $5^{\prime}$ GTCTAGCGAGAGCGTAACGG $3^{\prime}$ and 5' GCGGAGGCGGGACAAAGATGGG 3'.

\section{Construction of $\mathrm{Sp} 1$ expression plasmids}

Total RNA was obtained from human HeLa cells and reversetranscribed with M-MLV Reverse Transcriptase primed by oligo $(\mathrm{dT})_{15}$. Primers used in the subsequent PCR amplification of Spl cDNA were: forward, 5- GAAGCTTATGAGCGACGAAGATCACTCGATG-3, and reverse, 5-GGAATTCTCAGAAGGCATTGCGACTGA-3. The PCR products were digested with HindIII and EcoRI and inserted into pCDNA3.1(+).

A truncated form of $\mathrm{Spl}(\Delta \mathrm{Spl})$ lacking its DNA-binding domain and consisting of three zinc-fingers (amino acids 621-708) [8] was obtained by PCR-mediated deletion by the following sequence: 5'-CAGAATAAGAAGGGAGGCGCA-3' and 5'-AGGATCGCCGGAGCGCGTTCG-3'. All constructs were confirmed by DNA sequencing.

\section{RNA interference and real-time PCR}

One day before transfection, HFL1 cells were plated at a density of $5 \times 10^{4}$ cells per well in 24-well plates, then transfected with $20 \mathrm{nM}$ of Control siRNA (sc-37007, Santa Cruz Biotechnology, Inc.) or human SP1-specific siRNA (sc-29487, Santa Cruz Biotechnology, Inc.) using the siRNA Reagent System (sc-45064, Santa Cruz Biotechnology, Inc.) according to the manufacturer's instructions. After 24 hours, the medium was changed and the cells were further transfected with $0.2 \mu \mathrm{g}$ of a p-210/+52 construct using lipofectamine 2000 and incubated for another $24 \mathrm{~h}$. Luciferase assays were performed following the manufacturer's instructions. In parallel, wells were harvested after siRNA transfection. Total RNA was isolated from cells using TRIzol Reagent (Invitrogen), and reverse-transcribed as above. Real-time PCR was performed using SYBR Green PCR Master Mix (Takara Bio) on an ABI 7500 Real-Time PCR System (Applied Biosystems). USP22 primer pairs were as follows: forward, 5' GTGTCTTCTTCGGCTGTTTA -3', reverse, 5'CTCGTCGTTGGGGATTATTT-3'. Spl primer pairs were: forward, 5'-GCGGCTCCCAACTTACAGAA-3'; reverse, 5' TGCCTCGACTTCCTCGATTT- 3'

\section{Statistical analysis}

Data are presented as the mean \pm SEM. Statistical differences between sample means were determined using unpaired, two- 
tailed Student's $t$ test. Significance was set at a probability value less than 0.05 .

\section{Results}

\section{Identification of the 5 '-flanking region of the human USP22 gene}

The transcription initiation site(s) for USP22 have not yet been confirmed. We performed 5 ' - RACE to amplify the $5^{\prime}$-end of the USP22 cDNA from the HeLa cell mRNA. After reverse transcription and nested PCR, a band of approximately $260 \mathrm{bp}$ was obtained and then cloned into a pMD18-T vector. The sequencing results from 25 randomly selected clones revealed that a single transcriptional start site was located at 176 bp upstream of ATG in mature USP22 mRNA (Fig. 1A), which is identical to the USP22 genomic sequence (Fig. 1B). Sequence analysis showed that the region upstream of the transcriptional start site includes one GG box but lacks typical TATA boxes.

Based upon our findings regarding the transcriptional start site, we amplified by PCR from genomic DNA from HeLa cells a 2880-bp DNA fragment containing the region of the transcriptional start site, and inserted it into pMD-18T vectors for DNA sequencing. Surprisingly, we found two novel single-nucleotide polymorphisms (SNP) in the clone that are not documented in the existing human genome sequence in NCBI. The sequence from HeLa cells contained two mutations; T-G and T-G, at positions 283 and 280 upstream (positions -283 and -280 , respectively) of the transcriptional start site (Fig. 1C). Genomic DNA from an additional five healthy Chinese were isolated to detect these SNPs. Results showed that the two SNPs were present in all of the genomic DNAs.

To analyze the promoter activity of the 5 -flanking region of USP22, various truncated versions of the USP22 promoter were amplified and cloned into the luciferase reporter vector pGL-3 Basic. These constructs were transfected into human lung fibroblast (HFL1) or human tumor cells $(\mathrm{HeLa})$ to determine the sequence elements required for USP22 promoter activity. As shown in Fig. 2, the longest construct, p-2828/+52, was able to drive expression of the luciferase reporter gene in HFLl cells one hundred times higher than the pGL3-Basic construct, which indicates the transcriptional functionality of the promoter. In human tumor cells (HeLa), transfection with this construct also resulted in significantly higher activity (300 times greater than for
pGL3-Basic). The 3'-truncated construct p-2828/+1306, lacking the 3 '-end 1522 bp from P-2828/+52, displayed dramatically inhibited promoter activity in both HFLl and HeLa cells, indicating that the region -1306 to +52 was essential in driving the USP22 promoter. To further characterize the p-1306/+52 region, sequential 5'-deletion constructs from p-1306 were investigated. In HFL1 cells, the construct $\mathrm{p}-595 /+52$ showed the highest activity among the USP22 promoter constructs, which was approximately 300 times higher than the activity shown by pGL3Basic. The longer constructs, p-866/+52 and p-1306/+52, showed only $20 \%$ of the activity shown by $\mathrm{p}-595 /+52$, suggesting that the core promoter is present in the -595 to +52 regions, and that the sequence between -596 and -866 might contain a negative element(s) inhibiting promoter activity. The luciferase activity of p$326 /+52$ and $\mathrm{p}-210 /+52$ was approximately two-fold greater than that of $\mathrm{p}-2828 /+52$, but $30 \%$ lower than that of $\mathrm{p}-595 /+52$. The shortest construct, $\mathrm{P}-7 /+52$, demonstrated no luciferase activity. These data indicate that the promoter region between -210 and -7 is required for basal transcriptional activity of the USP22 gene, but further analysis is required.

In HeLa cells, the construct p-595/+52 showed approximately 900 times higher activity than pGL3-Basic, which was also the highest activity among all of the constructs. Similarly, the construct p-210/+52 was the shortest fragment exhibiting a high promoter activity, showing 700 times higher activity than pGL3Basic.

Mutations in the Sp1-binding site of the USP22 promoter lead to elevated transcription

Since the region -210 to -7 is required for basal transcriptional activity of the USP22 gene, we analyzed the putative transcriptional factor binding sites in this region using MatInspector software. As shown in Fig. 3A, this region contains a Splbinding site, a CREB/ATF binding site, and a c-Myb binding site. Among these motifs, the Spl-binding site attracted our attention because this site and the transcriptional start site are juxtaposed, and Spl has been reported to activate promoters lacking a TATA box. To evaluate the significance of the Sp1-binding site for USP22 transcription, we substituted GATGGG for the consensus sequence GGGCGG or deleted this consensus sequence in the parental vector $\mathrm{p}-210 /+52$ to generate $\mathrm{p}-210 /$ Splmut or $\mathrm{p}-210 /$ Spldel constructs, respectively. After transient transfection, the p210/Splmut and p-210/Spldel constructs showed an approxi-

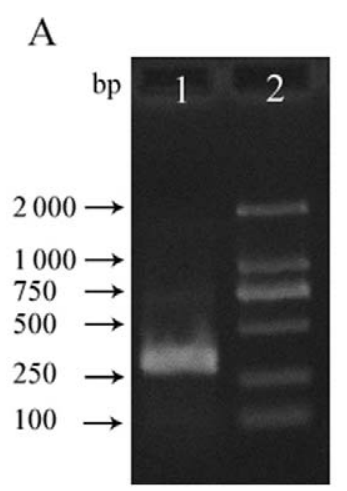

B Transeription start site ...... CTTGCAGCCTCCCCTCGGCGATCGCGCAGCCCCATCTTTGTCCGGCC TCCGCGCTTTGTTCTCGGCGCCCGGGCCTTGGCCAGCCTGGCCAGCCGCCG AGCAGCCCCCACGCCGCGCTGGCGTCGTCCTCGCCTCCCTCGCCGCCGCCC CCCGCGCGCGGCCGGGCCTTGCCCCCCATGGTGTCC......

C

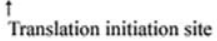

Sequence in NCBI: GGGGGGGGTGGCCTACTACCAATAAA

Sequence from HeLa G G G G G G G G GT G GC C G AC G ACC A A TA A A

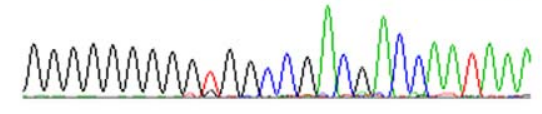

Figure 1. Identification of the transcriptional start site of the USP22 gene. A. Results of 5' RACE experiments on total RNA from HeLa cells; DNA sequences were detected by gel electrophoresis using 2\% agarose. Lane 1. A single DNA band of 260 bp was detected; Lane 2. DL2000 marker. B. Sequence of USP22 gene depicting the positions of the transcriptional start sites. C. Compared with the existing information in the human genome database, the DNA sequence of the HeLa clone contains two mutations, A-C and A-C, at positions 280 and 283 upstream from the transcriptional start site.

doi:10.1371/journal.pone.0052716.g001 


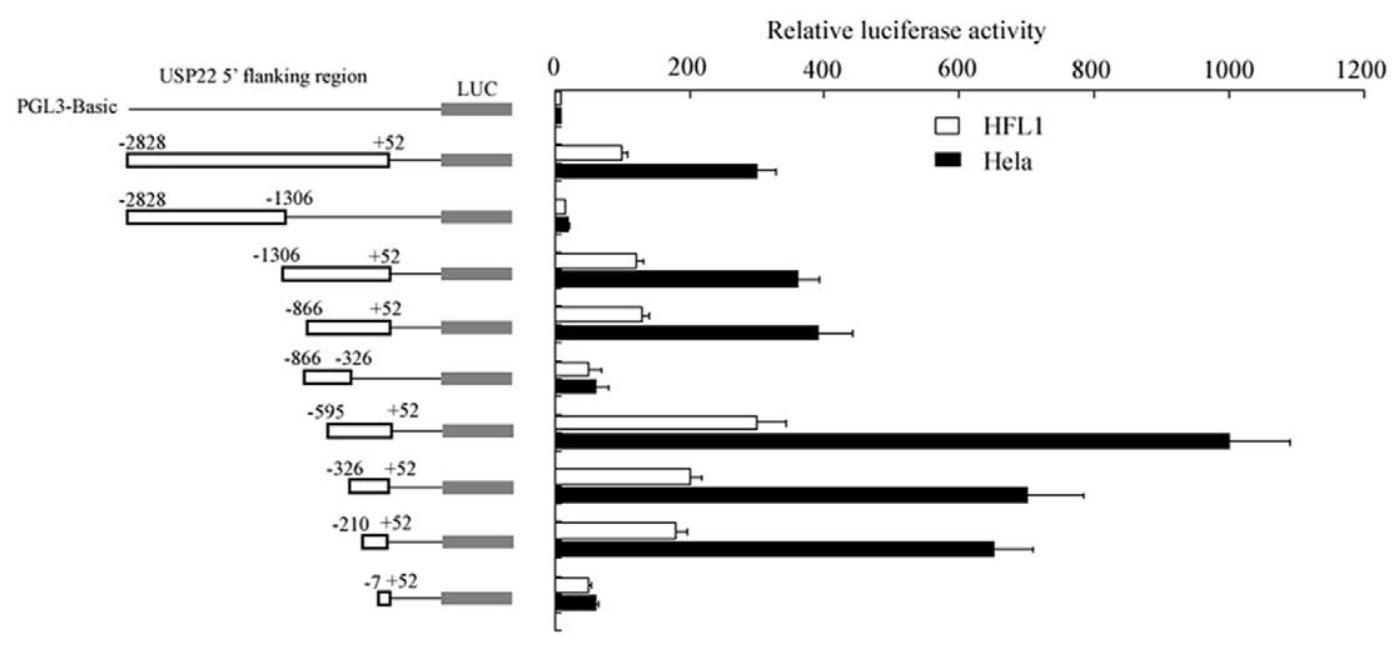

Figure 2. Luciferase reporter assay for the USP22 gene promoter. A series of fragments of the $5^{\prime}$-flanking USP22 promoter region is schematized. Each promoter-reporter construct or the promoter-less plasmid pGL3-basic, was co-transfected with pRL-TK into HFL1 and HeLa cells. Luciferase activities were measured after $24 \mathrm{~h}$ and normalized for transfection efficiency. The luciferase activity of each construct is presented relative to the pGL3-basic activity.

doi:10.1371/journal.pone.0052716.g002

mately $170 \%$ higher luciferase activity in HFLl cells compared with the p-210/+52 construct, suggesting that this DNA motif plays a negative role toward USP22 promoter activity (Fig. 3 B).

\section{Interaction between Sp1 and the USP22 Promoter}

Transcription factor Spl has a high affinity for the GC box; we therefore determined whether cellular Spl binds to this DNA motif in the USP22 promoter region. A ChIP assay was carried out using an antibody against human Spl. Immunoprecipitation of cross-linked chromatin from HFLl cells with an anti-Spl antibody followed by PCR amplification of the region (the sequence between-210 and +52) confirmed that the endogenous Spl protein does bind to this region of the USP22 promoter in HFLl (Fig. 4A).

Furthermore, we used the cell-permeable agent mithramycin A to inhibit the binding of Spl to DNA, since mithramycin A binds to GC-rich DNA sequences, thereby precluding the DNA binding of Spl [9]. After HFLl cells were transfected with a p-210/+52 plasmid and treated with $10 \mu \mathrm{M}$ mithramycin A for 12 hours, the luciferase activity was significantly higher compared with that in vehicle-treated cells (Fig. 4B).

\section{Effect of Sp1 on USP22 promoter activity}

Since HeLa cells showed high USP22 expression, we examined the effect of $\mathrm{Sp} 1$ on USP22 transcriptional regulation by cotransfecting HeLa cells with the p-210/+52 construct and a CMVdriven Spl expression vector. Forced expression of exogenous Spl repressed USP22 promoter activity by $40 \%$. The decline in USP22 promoter activity was not observed in the $\mathrm{p}-210 / \mathrm{Sp} 1 \mathrm{mut}$ construct lacking the $\mathrm{Spl}$ site. Additionally, we constructed a truncated form of $\mathrm{Spl}(\Delta \mathrm{Spl})$ lacking its DNA-binding domain and which consists of three zinc-fingers [10]. HeLa cells were transfected with either full-length $\mathrm{Spl}, \Delta \mathrm{Spl}$, or mock vector and

A
-210 GTCTACCCAGAGCCTAACGGGCGCGCGAGTGTGGGGGAAGGGCCTGGCGCGGGCTGG
-153 AGACTGCGCAGTGCGGTGCCGGCCGGGAGGGGGGGGGGGGGTGACGTAATCTCCGTC

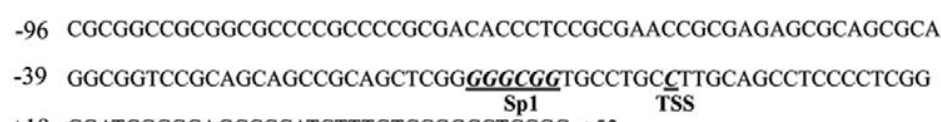
+19 CGATCGCGCAGCCCCATCTTTGTCCGGCCTCCGC +52 TSS

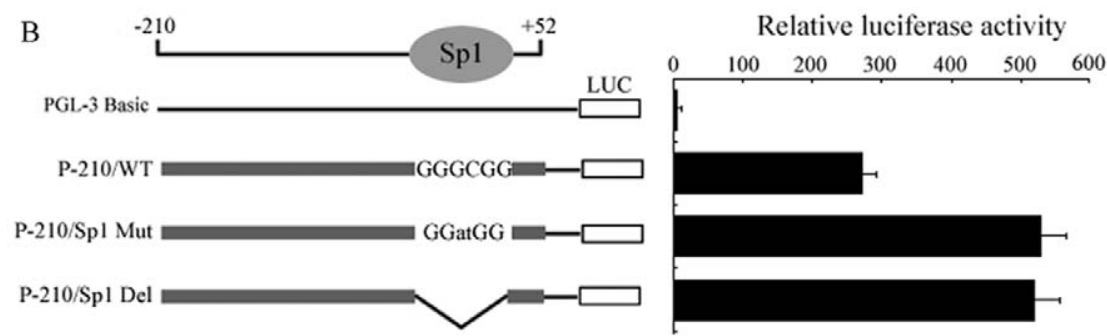

Figure 3. Mutation analyses of the Sp1-binding site. A. Nucleotide sequence and structural organization of the USP22 gene core promoter region. Putative binding sites for the transcriptional factors are underlined. B. Luciferase activity expressed by the Sp1 site-directed mutant and deletion mutants relative to pGL3-basic activity.

doi:10.1371/journal.pone.0052716.g003 

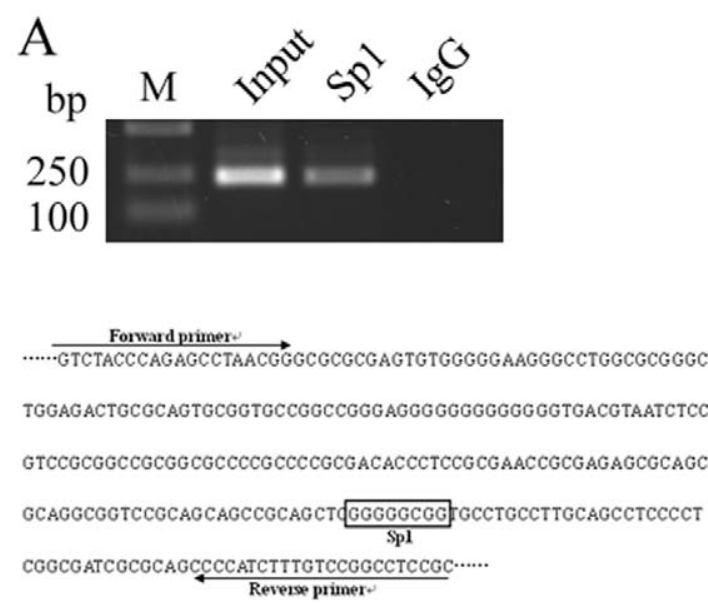

B
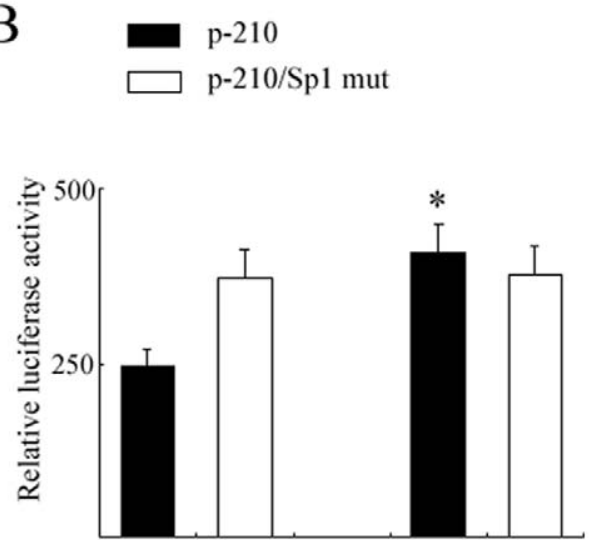

Mithramycin A
$(0 \mu \mathrm{M})$
$(10 \mu \mathrm{M})$

Figure 4. Determination of Sp1 binding to the USP22 gene promoter. A. DNA was isolated from HFL1 cells in each group and immunoprecipitated with antibodies against Sp1, RNA polymerase II or nonspecific rat IgG. Input and immunoprecipitated DNAs were then PCR amplified using primer pairs covering the Sp1-binding site. B. HFL1 cells expressing p-210 or p-210/Sp1mut constructs were treated with 10 um of mithramycin A or vehicle. Luciferase activity was determined $24 \mathrm{~h}$ later $(*, p<0.05$ vs. vehicle treatment). doi:10.1371/journal.pone.0052716.g004

examined 24 hours later. As expected, $\triangle \mathrm{Spl}$ transfection did not affect the USP22 promoter activity (Fig. 5A).

To investigate whether reduction of $\mathrm{Spl}$ protein can enhance USP22 promoter activity, we down-regulated Spl expression by RNA interference (RNAi). HFLl cells were co-transfected for 24 hours with siRNA that specifically targeted Spl and P-210/ +52 . As shown in Fig. 5B, we achieved about a $70 \%$ reduction in Spl protein, and the luciferase activity was significantly increased in siRNA-transfected HFLl cells; however no change in detectable luciferase activity was observed in the control siRNA-transfected cells. To determine the effect of $\mathrm{Spl}$ on endogenous USP22 expression, the mRNA levels of USP22 were detected by real-time PCR after siRNA transfection. As shown in Fig. 5C, the siRNAmediated knockdown of $\mathrm{Spl}$ led to a highly significant induction of USP22 mRNA compared with controls (over 1.8 fold).

\section{Discussion}

In this study, we identified and characterized the human USP22 promoter and its activity. For the first step, a single transcriptional initiation site of the human USP22 gene was confirmed. The sequence between the transcriptional initiation site and translational start site is identical to the USP22 DNA sequence, suggesting that this region is a major transcriptional start site of the human USP22 gene. Because the USP22 gene is abnormally activated in various human tumor cells [7], its promoter activity was investigated in both cultured HFL1 and HeLa cell lines. Using a luciferase reporter system, we found that the promoter constructs of USP22 displayed relatively higher activity in HeLa cells than in HFL1, cells indicating abnormal transcriptional activity of the USP22 gene in human tumor cells. By generating a series of $5^{\prime}$ deletions, we confirmed that the region between -210 and -7 is required for the basal activity of the USP22 promoter both in HFL1 and HeLa cells; we therefore focused our attention on this region. Bioinformatics analysis shows that, within this region, there are putative binding sites for transcription factors Spl, CREB/ ATF, and Myb. Among these, a single Spl-binding site located at -7 to -13 of the USP22 promoter drew our attention. Unexpectedly, mutation or deletion of this motif resulted in a significant enhancement in USP22 promoter activity, suggesting that the Spl-binding site functions as a negative element for USP22 gene transcription.

The transcription factor Spl binds to various promoters with high affinity, and acts as an activator or an inhibitor depending upon the genes $[11,12,13]$. Accumulating evidence shows that Spl plays a role in tumorigenesis. For example, Spl activity is increased following phosphorylation by oncogenic signaling pathways such as RAS/RAF/MAPK and PI3K/Akt [14,15,16]. In addition, $\mathrm{Spl}$ is overexpressed or hyperactivated in a number of human tumors $[17,18]$. Multiple genes involved in tumorigenesis, such as those in cell growth, apoptosis and angiogenesis, are found to be promoted by Sp1, including survivin [19], VEGF [15] and NME5 [20]. Based on these findings, we initially hypothesized that Spl might play a positive role in USP22 gene transcription, since USP22 is up-regulated in most human tumor cells. However, the experimental results do not support our hypothesis. The binding of Spl to the DNA motif juxtaposed with the transcriptional start site (which consequently prevents transcriptional initiation), might be a mechanism for Spl's negative role in USP22 transcription. Another possibility is the formation of secondary structures caused by the high GC content, leading to a reduced transcriptional level.

To verify these assumptions, we performed ChIP assays. Results showed that Spl binds to the USP22 promoter in fibroblasts, suggesting that Spl-DNA interaction may be required for USP22 repression. We next performed several experiments on different aspects to further verify this hypothesis. First, we inhibited Spl binding activity with mithramycin A. When HFL1 cells expressing the construct p-210/+52 were treated with mithramycin A for $12 \mathrm{~h}$, luciferase activity was significantly increased compared with that of vehicle-treated cells. Second, forced expression of Spl decreased p-210/+52 luciferase activity; however, the truncated Spl lacking its DNA-binding domain did not affect luciferase activity. Third, the knock-down of Sp1 expression induced USP22 promoter activity. Finally, we confirmed that over-expression of Spl inhibited the endogenous USP22 expression in human tumor cells. Collectively, these results strongly indicate that $\mathrm{Spl}$ plays a negative role and that its DNA-binding activity is a key step needed for USP22 transcription.

Although Spl has been described primarily as a transcriptional activator, recent results show that $\mathrm{Spl}$ also plays a role in 
A $\mathrm{p}-210$ $\mathrm{p}-210 / \mathrm{Sp} 1 \mathrm{mut}$

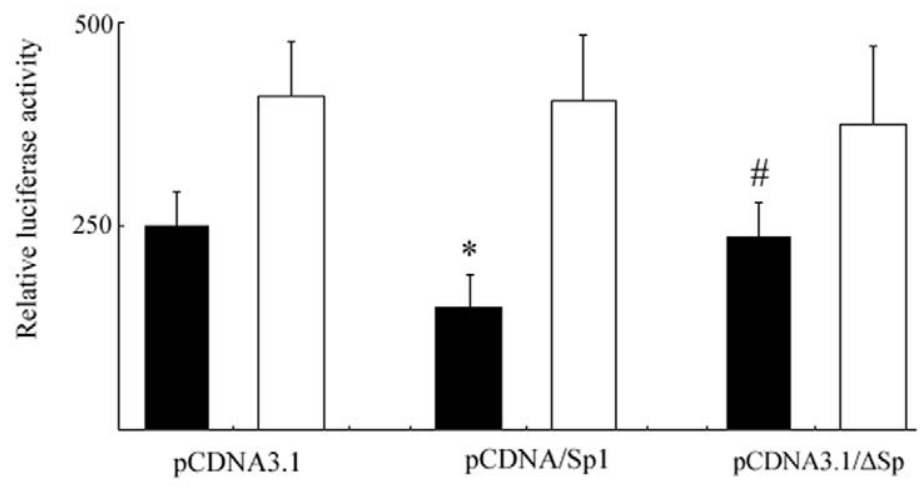

B

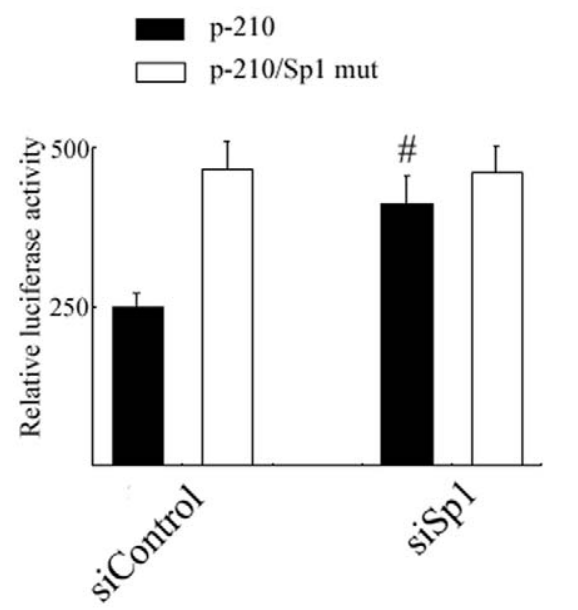

$\mathrm{C}$

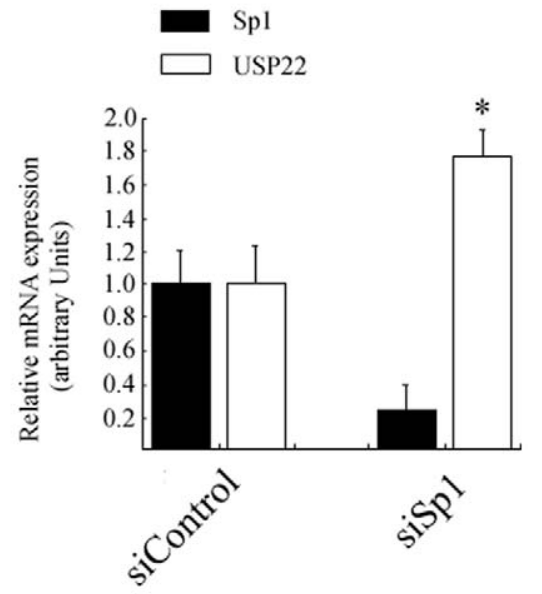

Figure 5. Effects of Sp1 on the USP22 promoter. A. HeLa cells were co-transfected with USP22 promoter plasmids $p-210$ or $p-210 / S p 1$ mut and expression plasmids for either Sp1, $\Delta S p$ or an empty vector (pCDNA3.1). Luciferase activity was determined $24 \mathrm{~h}$ later. Luciferase activities are expressed as the percentage of $p-210$ relative to $p C D N A 3.1$ activity. $(*, p<0.05$ vs. pCDNA3.1; \#, $p>0.05$ vs. pCDNA3.1). B. HFL1 cells were transfected with Sp1 siRNA or non-targeting control siRNA. Sp1 and USP22 mRNA expression was determined by real-time PCR ${ }^{*}, p<0.05$ vs. siControl). C. HFL1 cells were co-transfected with $\mathrm{p}-210$ or p-210/sp1 mut and Sp1 siRNA or non-targeting control siRNA, and with pRL-RK. Luciferase activities are expressed as the percentage of $p-210$ activity in the presence of control siRNA (\#, $p<0.05$ vs. siControl). doi:10.1371/journal.pone.0052716.g005

transcriptional repression in multiple promoters [13,21,22]. Spl represses gene expression via protein-protein interaction, or interplay with other transcription factors such as $\mathrm{Rb}$ [13] or p53 [23]. However, we did not identify any partner protein that cooperates with $\mathrm{Sp} 1$ to repress USP22 expression; this remains to be elucidated in future work. Conversely, Spl binding to DNA is reversible and adjustable. Growing evidence indicates that the posttranslational modifications of Spl protein (such as phosphorylation [24], acetylation [25], sumoylation [26], and glycosylation [27]), can influence its DNA-binding ability and gene transactivation. Understanding whether or how these posttranslational modifications influence Spl-DNA interaction is crucial for uncovering USP22 transcription mechanisms.
For some time $\mathrm{Spl}$ protein expression was believed to be a critical factor in tumor development, growth and metastasis, but other studies argue that its over-expression is detrimental to various cells. This controversy indicates an intricate role for $\mathrm{Spl}$ in cellular physiology. How Spl induces cell apoptosis is poorly understood currently. Multiple mechanisms are probably involved in Spl-induced apoptosis. For example, the $B c l-x$ gene (which encodes an anti-apoptotic protein), was suppressed in Sploverexpressing Baf-3 cells [8]. P53 was also found to accumulate in Sp1- overexpressing cancer cells [28]. In addition to these proposed mechanisms, our studies indicate that the increased Spl expression may, via binding to the promoter, contribute to the inhibition of USP22 expression, thus inducing cell-cycle arrest. 
In conclusion, we have cloned and characterized the proximal human USP22 promoter. Our data demonstrate for the first time that the transcription factor $\mathrm{Spl}$ is involved in the negative regulation of human USP22 expression. These findings provide new insights into Spl's function and regulation of USP22 transcription

\section{References}

1. Lee HJ, Kim MS, Shin JM, Park TJ, Chung HM, et al. (2006) The expression patterns of deubiquitinating enzymes, USP22 and Usp22. Gene Expr Patterns 6: $277-284$.

2. Zhang XY, Varthi M, Sykes SM, Phillips C, Warzecha C, et al. (2008) The putative cancer stem cell marker USP22 is a subunit of the human SAGA complex required for activated transcription and cell-cycle progression. Mol Cell 29: $102-111$.

3. Atanassov BS, Dent SY (2011) USP22 regulates cell proliferation by deubiquitinating the transcriptional regulator FBP1. EMBO Rep 12: 924-930.

4. Atanassov BS, Evrard YA, Multani AS, Zhang Z, Tora L, et al. (2009) Gcn5 and SAGA regulate shelterin protein turnover and telomere maintenance. Mol Cell 35: $352-364$.

5. Ovaa H, Kessler BM, Rolen U, Galardy PJ, Ploegh HL, et al. (2004) Activitybased ubiquitin-specific protease (USP) profiling of virus-infected and malignant human cells. Proc Natl Acad Sci U S A 101: 2253-2258.

6. Glinsky GV (2005) Death-from-cancer signatures and stem cell contribution to metastatic cancer. Cell Cycle 4: 1171-1175.

7. Glinsky GV (2006) Genomic models of metastatic cancer: functional analysis of death-from-cancer signature genes reveals aneuploid, anoikis-resistant, metastasis-enabling phenotype with altered cell cycle control and activated Polycomb Group (PcG) protein chromatin silencing pathway. Cell Cycle 5: 1208-1216.

8. Deniaud E, Baguet J, Mathieu AL, Pages G, Marvel J, et al. (2006) Overexpression of Spl transcription factor induces apoptosis. Oncogene 25: 7096-7105.

9. Miller DM, Polansky DA, Thomas SD, Ray R, Campbell VW, et al. (1987) Mithramycin selectively inhibits transcription of G-C containing DNA. AmJ Med Sci 294: 388-394.

10. Philipsen S, Suske G (1999) A tale of three fingers: the family of mammalian Sp/ XKLF transcription factors. Nucleic Acids Res 27: 2991-3000.

11. Kim S, Kang JK, Kim YK, Seo DW, Ahn SH, et al. (2006) Histone deacetylase inhibitor apicidin induces cyclin $\mathrm{E}$ expression through $\mathrm{Spl}$ sites. Biochem Biophys Res Commun 342: 1168-1173.

12. Banchio C, Schang LM, Vance DE (2003) Activation of CTP:phosphocholine cytidylyltransferase alpha expression during the $\mathrm{S}$ phase of the cell cycle is mediated by the transcription factor Spl. J Biol Chem 278: 32457-32464.

13. Law AY, Yeung BH, Ching LY, Wong CK (2011) Spl is a transcription repressor to stanniocalcin-1 expression in TSA-treated human colon cancer cells, HT29. J Cell Biochem 112: 2089-2096.

14. Milanini-Mongiat J, Pouyssegur J, Pages G (2002) Identification of two Spl phosphorylation sites for $\mathrm{p} 42 / \mathrm{p} 44$ mitogen-activated protein kinases: their

\section{Acknowledgments}

We thank Dr. Zhendong Zhong for data analysis and writing assistance.

\section{Author Contributions}

Conceived and designed the experiments: WDL JJX. Performed the experiments: JJX XXG XQL. Analyzed the data: HY ZG. Contributed reagents/materials/analysis tools: HY ZG. Wrote the paper: HY.

implication in vascular endothelial growth factor gene transcription. J Biol Chem 277: 20631-20639.

15. Pore N, Liu S, Shu HK, Li B, Haas-Kogan D, et al. (2004) Spl is involved in Akt-mediated induction of VEGF expression through an HIF-1-independent mechanism. Mol Biol Cell 15: 4841-4853.

16. Chuang CW, Pan MR, Hou MF, Hung WC (2012) Cyclooxygenase-2 upregulates CCR7 expression via AKT-mediated phosphorylation and activation of Sp1 in breast cancer cells. J Cell Physiol 228(2): 341-348.

17. Hsu TI, Wang MC, Chen SY, Yeh YM, Su WC, et al. (2011) Sp1 expression regulates lung tumor progression. Oncogene 568: 1-16.

18. Wang L, Wei D, Huang S, Peng Z, Le X, et al. (2003) Transcription factor Sp1 expression is a significant predictor of survival in human gastric cancer. Clin Cancer Res 9: 6371-6380.

19. Liu YL, Jiang SX, Yang YM, Xu H, Liu JL, et al. (2012) USP22 Acts as an Oncogene by the Activation of BMI-1-Mediated INK4a/ARF Pathway and Akt Pathway. Cell Biochem Biophys 62: 229-235.

20. Li F, Jiang Z, Wang K, Guo J, Hu G, et al. (2012) Transactivation of the human NME5 gene by Sp1 in pancreatic cancer cells. Gene 503: 200-207.

21. Mottet D, Pirotte S, Lamour V, Hagedorn M, Javerzat S, et al. (2009) HDAC4 represses $\mathrm{p} 21$ (WAF1/Cipl) expression in human cancer cells through a Sp1dependent, p53-independent mechanism. Oncogene 28: 243-256.

22. Cole LK, Vance DE (2010) A role for Spl in transcriptional regulation of phosphatidylethanolamine N-methyltransferase in liver and 3T3-L1 adipocytes. J Biol Chem 285: 11880-11891.

23. Dalvai M, Mondesert O, Bourdon JC, Ducommun B, Dozier G (2011) Cdc25B is negatively regulated by p53 through $\mathrm{Spl}$ and NF-Y transcription factors. Oncogene 30: 2282-2288.

24. Guo L, Eviatar-Ribak T, Miskimins R (2010) Spl phosphorylation is involved in myelin basic protein gene transcription. J Neurosci Res 88: 3233-3242.

25. Waby JS, Chirakkal H, Yu G, Griffiths GJ, Benson RS, et al. (2010) Spl acetylation is associated with loss of DNA binding at promoters associated with cell cycle arrest and cell death in a colon cell line. Mol Cancer 9: 275.

26. Spengler ML, Brattain MG (2006) Sumoylation inhibits cleavage of Spl Nterminal negative regulatory domain and inhibits Spl-dependent transcription. J Biol Chem 281: 5567-5574

27. Vij N, Zeitlin PL (2006) Regulation of the ClC-2 lung epithelial chloride channel by glycosylation of SP1. Am J Respir Cell Mol Biol 34: 754-759.

28. Chuang JY, Wu CH, Lai MD, Chang WC, Hung JJ (2009) Overexpression of Sp1 leads to p53-dependent apoptosis in cancer cells. Int J Cancer 125: 20662076 